\title{
LITTLEWOOD-PALEY THEORY ON GAUSSIAN SPACES
}

\author{
JÜRGEN POTTHOFF*)
}

\section{§1. Introduction}

In this article we prove a number of inequalities of Littlewood-PaleyStein (LPS) type for functions on general Gaussian spaces (s. below).

In finite dimensional Euclidean spaces (with Lebesgue measure) the power of such inequalities has been demonstrated in Stein's book [12]. In his second book [13], Stein treats other spaces too: also the situation of a general measure space $(X, \mu)$. However the latter case is too general to allow for a rich class of inequalities (cf. Theorem 10 in [13]).

Meyer has proved in [6] a large set of LPS inequalities for the Wiener space, i.e. larger than in Stein's theorem quoted above. This is of course possible due to the concrete nature of the Wiener measure. As an application, Meyer has proved in [7] an equivalence of certain norms on Wiener space and the algebraic structure of a space of "smooth" Wiener functionals. These results have in turn important applications in the Malliavin calculus. The generalizations of these results for general Gaussian spaces will be discussed in a forthcoming paper.

Here we consider the following situation: let $\mathscr{N}$ be a real, separable, nuclear pre-Hilbert space with scalar product $(\cdot, \cdot)$, compatible with the nuclear topology of $\mathscr{N}$. $\mathscr{H}$ denotes the completion of $\mathscr{N}$ in the norm $|\cdot|$ induced by $(\cdot, \cdot)$, and $\mathscr{N}^{*}$ is the (topological) dual of $\mathscr{N}$. By $\mathscr{B}$ we denote the $\sigma$-algebra generated by the cylinder sets of $\mathscr{N}^{*}$ and $d_{\mu}$ is the Gaussian measure on $\mathscr{N}$ defined by the scalar product on $\mathscr{H}$ (via the Bochner-Minlos theorem [3, 5]):

$$
\int_{\mathscr{N}^{*}} \exp (i\langle x, \xi\rangle) d \mu(x)=\exp \left(-\frac{1}{2}|\xi|^{2}\right)
$$

for $\xi \in \mathscr{N},\langle\cdot, \cdot\rangle$ denoting the pairing of $\mathscr{N}^{*}$ and $\mathscr{N}$. We refer to the triple $\left(\mathscr{N}^{*}, \mathscr{B}, d \mu\right)$ as a Gaussian space.

Received April 7, 1986.

*) Supported in part by Japan Society for the Promotion of Science. 
As we shall see, this structure is rich enough to obtain a large class of LPS-inequalities, including the one of Meyer. (Note that Wiener functionals can be represented as white noise functionals, i.e. functions on $\mathscr{N}^{*}=\mathscr{S}^{*}(\boldsymbol{R})$ and $\mathscr{H}$ is chosen as $L^{2}(\boldsymbol{R}, d t)$, cf. also [5]). Moreover the framework described above is most fitting for the discussion of constructive quantum field theory and applications of the inequalities presented here in this field are expected.

Meyer proves his set of inequalities by martingale methods, while in this paper we shall use techniques from Fock space calculus $[2,8,9,10,11]$ and functional analysis. The strategy of proof is adopted from Stein's proof in [13] for compact Lie groups.

Roughly speaking the inequalities to be shown fall into two classes: "parabolic" inequalities and "elliptic" inequalities.

To explain these notions, consider $L^{2} \equiv L^{2}\left(\mathscr{N}^{*}, d \mu\right)$. It is well-known (e.g. $[9,11])$ that $L^{2}$ is isomorphic to the symmetric Fock space $\Gamma(\mathscr{H})$ over the complexification $\mathscr{H}_{c}$ of $\mathscr{H}$. If $A$ is a (densely defined, closed) linear operator on $\mathscr{H}$, then we have on $\Gamma(\mathscr{H})$ the (densely defined, closable) linear operators $\Gamma(A)$ and $d \Gamma(A)$, the so-called second quantizations of $A$ (cf. $[2,9,11])$. The corresponding isometric images of these operators on $L^{2}$ will be denoted by the same symbols. If $A$ is selfadjoint on $\mathscr{H}$ with domain $\mathscr{D}(A)$, then $\Gamma(A)$ and $d \Gamma(A)$ are essentially selfadjoint on a domain, which can be explicitly constructed from $\mathscr{D}(A)$ [2]. Assume that $A$ is selfadjoint and positive, then $\Gamma(A)$ and $d \Gamma(A)$ are positive. We are going to consider the semigroups $\left\{\boldsymbol{P}_{t}, t \in \boldsymbol{R}_{+}\right\},\left\{\boldsymbol{Q}_{t}, t \in \boldsymbol{R}_{+}\right\}$with

$$
\begin{aligned}
& P_{t}:=\exp (-t d \Gamma(A)), \\
& Q_{t}:=\exp \left(-t(d \Gamma(A))^{1 / 2}\right) .
\end{aligned}
$$

For $h \in L^{2}$ consider then $P_{t} h$ and $Q_{t} h$, which satisfy a "parabolic", "elliptic" resp. equation:

$$
\begin{aligned}
& \left(\partial_{t}+d \Gamma(A)\right) P_{t} h=0, \\
& \left(\partial_{t}^{2}-d \Gamma(A)\right) Q_{t} h=0 .
\end{aligned}
$$

These equations will play a crucial role in the estimates we are going to prove, therefore the above mentioned names.

For the rest of this paper, we make the following assumptions on $A$ : (A.1) $A$ is selfadjoint on $\mathscr{H}$ and $A \geq 1$ 
(A.2) $A$ admits a complete set of eigenvectors $\left\{e_{k}, k \in Z_{+}\right\}, A e_{k}=\lambda_{k} e_{k}$, which belong to $\mathscr{N}$.

Unfortunately, the rather restrictive condition (A.2) has to be posed for some technical reason and it would be interesting to see, if, with another strategy of proof than given here, it can be relaxed. Two typical examples are: $A=1$, so that $d I^{\prime}(A)$ is the number operator, or OrnsteinUhlenbeck operator; $A=-\Delta+|x|^{2}$ for $\mathscr{N}=\mathscr{S}\left(\boldsymbol{R}^{d}\right), \mathscr{H}=L^{2}\left(\boldsymbol{R}^{d}, d x\right)$.

Let us now introduce the following three Littlewood-Paley functions:

$$
\begin{aligned}
& G_{1}(f):=\left(\int_{0}^{\infty}\left(P_{t} \mid \nabla P_{t} f\right)^{2} d t\right)^{1 / 2} \\
& G_{2}(f):=\left(\int_{0}^{\infty} P_{t}\left|\nabla P_{t} f\right|^{2} d t\right)^{1 / 2} \\
& G_{3}(f):=\left(\int_{0}^{\infty} t\left[\left|\nabla Q_{t} f\right|^{2}+\left|\partial_{t} Q_{t} f\right|^{2}\right] d t\right)^{1 / 2}
\end{aligned}
$$

where

$$
|\nabla g|^{2}:=\sum_{k=0}^{\infty} \lambda_{k}\left|\partial\left(e_{k}\right) g\right|^{2}
$$

and $\partial(\eta), \eta \in \mathscr{H}_{c}$, is the isometric image of the Fock space annihilation operator on $L^{2}$ (both denoted by the same symbol), cf. e.g. [10, 11], normalized in such a way, so that it becomes a derivation. (More precisely on Fock space

$$
\Gamma(\mathscr{H})=\bigoplus_{n=0}^{\infty}\left(\mathscr{H}_{c}\right)^{\hat{\otimes}^{n}}
$$

$\hat{\otimes}$ being the symmetric tensor product, $\partial(\eta)$ acts on elements $F=f_{1} \hat{\otimes} \ldots$ $\hat{\otimes} f_{n}$ via

$$
\partial(\eta) F=\sum_{i=1}^{n}\left(\eta, f_{i}\right) \hat{\bigotimes}_{\substack{j=1 \\ j \neq \imath}}^{n} f_{j}
$$

and (1.11) is extended in the usual way $[10,11])$.

Furthermore we shall employ the following convenient notation: if there exists a strictly positive constant $c_{p, q}$, depending on $p$ and $q$ only, so that $\|f\|_{p} \leq c_{p, q}\|g\|_{q}$, we shall write $\|f\|_{p} \lesssim\|g\|_{q}$.

Now we are ready to state the main results.

Theorem A (Parabolic Case). Let $f \in L^{p} \equiv L^{p}(\mathscr{N}, d \mu)$, for some $p \in$ $(1, \infty)$. Then $G_{1}(f)$ is in $L^{p}$ too and 


$$
\left\|G_{1}(f)\right\|_{p} \lesssim\|f\|_{p}
$$

If $p \in[2, \infty)$, then also $G_{2}(f) \in L^{p}$, with

$$
\left\|G_{2}(f)\right\|_{p} \lesssim\|f\|_{p}
$$

If for some $p \in(1,2] G_{2}(f) \in L^{p}$ and $\int f d \mu=0$, then also $f \in L^{p}$ and

$$
\|f\|_{p} \lesssim\left\|G_{2}(f)\right\|_{p}
$$

Theorem B (Elliptic Case). Let $f \in L^{p}$, for some $p \in(1, \infty)$. Then $G_{3}(f)$ belongs to $L^{p}$ too and

$$
\left\|G_{3}(f)\right\|_{p} \lesssim\|f\|_{p}
$$

If furthermore $\int f d \mu=0$, then also the converse inequality holds

$$
\|f\|_{p} \lesssim\left\|G_{3}(f)\right\|_{p}
$$

In section 2 we shall prepare the proofs, recalling some properties of semigroups on $L^{p}$ and proving integral representations for $P_{t}$ and $Q_{t}$, which will provide some simple estimates, that we shall use frequently.

Section 3 will be concerned with the proof of Theorem A, while section 4 treats the elliptic case. Since the strategies of proof are quite parallel, section 4 is more sketchy, however it includes some useful corollaries of Theorem B.

Acknowledgement. The author acknowledges gratefully the warm hospitality of Prof. T. Hida and the Department of Mathematics, Nagoya University. It is a pleasure to thank Prof. T. Hida, Dr. T. Funaki, and N. Obata for discussions.

\section{§2. Semigroups on $L^{p}$}

Consider the semigroup $\left\{P_{t}, t \in R_{+}\right\}$on $L^{2}$ given by (1.2). Simon [11] shows that $\left\{P_{t}\right\}$ is a strongly continuous contraction semigroup on all $L^{p}, 1 \leq p \leq \infty$ and the formula

$$
\exp (-t d \Gamma(A))=\Gamma(\exp (-t A))
$$

For $\lambda \geq 0$ and $t>0$ we have $[4 ; 314,9 \mathrm{c}]$

$$
\exp \left(-t \lambda^{1 / 2}\right)=\int_{0}^{\infty} \exp (-s \lambda) \nu_{t}(d s)
$$


with

$$
\nu_{t}(d s)=(4 \pi)^{-1 / 2} t s^{-3 / 2} \exp \left(-t^{2} / 4 s\right) d s .
$$

Thus, by the spectral theorem, on $L^{p}, p \geq 2$

$$
Q_{t}=\int_{0}^{\infty} P_{s} \nu_{t}(d s)
$$

for all $t \in \boldsymbol{R}_{+}$.

From (2.3) we conclude that $Q_{t}$ too is a strongly continuous contraction semigroup on $L^{p}$, at least for $p \geq 2$, but by density of $L^{2}$ in $L^{1}$, this extends to all $p \geq 1$. Furthermore, since $P_{t}$ is positivity improving (cf. [11]) and $\nu_{t}(d s)$ is a positive measure on $\boldsymbol{R}_{+}$, also $Q_{t}$ is positivity improving.

For $x \in \mathscr{N}^{*}$ we define $\exp (-t A) x$ as the element of $\mathscr{N}^{*}$, which maps $\xi \in \mathscr{N}$ into $\langle x, \exp (-t A) \xi\rangle$ (this makes sense because of condition (A.2), section 1). Define

$$
: \exp (i\langle x, \xi\rangle)::=\exp \left(i\langle x, \xi\rangle+\frac{1}{2}|\xi|^{2}\right)
$$

for $x \in \mathscr{N}^{*}, \xi \in \mathscr{N}$ and denote by $\mathscr{E}$ the algebra generated from such functionals over $\mathscr{N}^{*}$, as $\xi$ ranges over $\mathscr{N}$.

A standard computation (e.g. using (2.1)) shows that

$$
P_{t}: \exp (i\langle x, \xi\rangle):=: \exp \left(i\left\langle e^{-t A} x, \xi\right\rangle\right):
$$

It is then easy to establish

Lemma 2.1. Let $f \in L^{p}, 1 \leq p<\infty$. Then for $t>0$

$$
\begin{gathered}
\left(P_{t} f\right)(x)=\int_{\mathcal{N}^{*}} f\left(e^{-t A} x+\left(1-e^{-2 t A}\right)^{1 / 2} y\right) d \mu(y), \\
\left(Q_{t} f\right)(x)=\int_{\mathcal{N}^{*}} \int_{0}^{\infty} f\left(e^{-s A} x+\left(1-e^{-2 s A}\right)^{1 / 2} y\right) \nu_{t}(d s) d \mu(y)
\end{gathered}
$$

hold in $L^{p}$-sense.

Sketch of the proof. Verify (2.6) on functions of the type (2.4). Next use the fact that $\mathscr{E}$ is dense in all $L^{p}, 1 \leq p<\infty$. (2.7) follows from (2.6) and (2.3).

Let us denote by $\mathscr{P}$ the algebra generated by polynomials in the variables $\left\langle x, e_{k}\right\rangle, k \in Z_{+}$(cf. (A.2), section 1). Note that $\mathscr{P}$ too is dense in all $L^{p}, 1 \leq p<\infty$. 
The following two lemmas are also easy to verify and the proofs are left to the reader.

Lemma 2.2. On $\mathscr{E}$ or $\mathscr{P}$

$$
\begin{aligned}
d \Gamma(A) & =\sum_{k} \lambda_{k} \partial^{*}\left(e_{k}\right) \partial\left(e_{k}\right) \\
& =\sum_{k} \lambda_{k}\left(-\partial\left(e_{k}\right)^{2}+\left\langle x, e_{k}\right\rangle \partial\left(e_{k}\right)\right)
\end{aligned}
$$

where $\partial^{*}\left(e_{k}\right)$ is the $L^{2}$-adjoint of $\partial\left(e_{k}\right)$.

Lemma 2.3. On $\mathscr{E}$ or $\mathscr{P}$ and for $\eta \in \mathscr{H}$

$$
\begin{aligned}
& P_{t} \partial\left(e^{-t A} \eta\right)=\partial(\eta) P_{t} \\
& P_{t} \partial^{*}(\eta)=\partial^{*}\left(e^{-t A} \eta\right) P_{t} .
\end{aligned}
$$

Also we shall make use of

Lemma 2.4. Let $f \in \mathscr{E}$ or $\mathscr{P}$ and $u \in[0, t]$. Then

$$
\left|\nabla P_{t} f\right|^{2} \leq e^{-2 u}\left(P_{u}\left|\nabla P_{t-u} f\right|\right)^{2} .
$$

Proof. Recall (1.9). By the semigroup property of $P_{t}$

$$
\begin{aligned}
\left|\nabla P_{t} f\right|^{2} & =\sum_{k} \lambda_{k}\left|\partial\left(e_{k}\right) P_{u} P_{t-u} f\right|^{2} \\
& =\sum_{k} \lambda_{k}\left|P_{u} \partial\left(e^{-u A} e_{k}\right) P_{t-u} f\right|^{2} \\
& =\sum_{k} \lambda_{k} e^{-2 u \lambda_{k}}\left|P_{u} \partial\left(e_{k}\right) P_{t-u} f\right|^{2} \\
& \leq e^{-2 u}\left(P_{u}\left|\nabla P_{t-u} f\right|^{2}\right)
\end{aligned}
$$

where we used Lemma 2.3 in the second equality. $\lambda_{k} \geq 1$ together with the integral representation (2.6) yields the last inequality, if we take the $l^{2}$-norm $|g|=.\left(\sum_{k}\left|g_{k}\right|^{2}\right)^{1 / 2}$ under the integral by convexity.

Corollary 2.5. For $f \in \mathscr{E}$ or $\mathscr{P}$

$$
\begin{aligned}
\left|\nabla P_{t} f\right|^{2} & \leq e^{-t} P_{t / 2}\left|\nabla P_{t / 2} f\right|^{2} \\
\left|\nabla Q_{t} f\right|^{2} & \leq e^{-t}\left(Q_{t / 2} \mid \nabla Q_{t / 2} f\right)^{2} \\
& \leq e^{-t} Q_{t / 2}\left|\nabla Q_{t / 2} f\right|^{2} .
\end{aligned}
$$

Proof. Lemmas (2.1), (2.4) and Schwarz' inequality.

\section{§ 3. LPS-Inequalities: Parabolic Case}

We start with two simple lemmas. 
LEMMA 3.1. Let $f$ be a pointwise defined, positive cylinder function on $\mathscr{N}^{*}$ in $L^{2}$, depending on finitely many variables $\left\langle x, e_{k}\right\rangle, k \in Z_{+}$. Then for all $p>1, t>0$

$$
\left(\partial_{t}+d \Gamma(A)\right)\left(P_{t} f\right)^{p}=-p(p-1)\left(P_{t} f\right)^{p-2}\left|\nabla P_{t} f\right|^{2} .
$$

Proof. First of all note that equation (3.1) makes sense, since $P_{t} f>0$ for $t>0$, because $P_{t}$ is positivity improving. Also the hypotheses on $f$ ensure that $\left(\partial_{t}+d \Gamma(A)\right)\left(P_{t} f\right)^{p}$ and $\left|\nabla P_{t} f\right|^{2}$ are well-defined. But then (3.1) is an easy consequence of a straightforward computation, using (1.4), (2.9) and the fact that $\partial(\eta)$ is a derivation.

LEMma 3.2. Let $\left\{F(\cdot, t), t \in \boldsymbol{R}_{+}\right\}$be a family of $L^{2}$-functions and assume that, as a function of $t, F(\cdot, t) \in C^{1}$ (in $L^{2}$-sense). Define

$$
I(x):=-\int_{0}^{\infty} P_{t}\left(\partial_{t}+d \Gamma(A)\right) F(x, t) d t .
$$

Then $I(x) \in L^{1}$ and

$$
\int I(x) d \mu(x)=\int(F(x, 0)-F(x, \infty)) d \mu(x) .
$$

Proof. Note that $d \Gamma(A) 1=0$ and $P_{t} 1=1$. Then selfadjointness of $P_{t}$ and $d \Gamma(A)$ on $L^{2}$, together with integration by parts in $d t$ prove (3.3), which in turn shows that $I \in L^{1}$. The following lemma gives a sense to definition (1.6) for $f \in L^{2}$ and $p \in(1,2]$

Lemma 3.3. For all $f \in L^{2}$ and $p \in(1,2], G_{1}(f) \in L$ and

$$
\left\|G_{1}(f)\right\|_{p} \lesssim\|f\|_{2} .
$$

Proof. It is sufficient to show (3.4) for $f \in \mathscr{P}$. Note, that in this case, $|\nabla f|$ involves only a finite number of terms and is pointwise defined (cf. (1.11)). Moreover

$$
G_{1}(f) \leq G_{2}(f)
$$

by Lemma 2.1 and Schwarz' inequality. Hence

$$
\left\|G_{1}(f)\right\|_{p} \leq\left\|G_{2}(f)\right\|_{p} \leq\left\|G_{2}(f)\right\|_{2}
$$

using Hölder's inequality. Now, for $f$ s.t. $\int f d \mu=0$

$$
\left\|G_{2}(f)\right\|_{2}=2^{-1 / 2}\|f\|_{2}
$$


because

$$
\left\|G_{2}(f)\right\|_{2}^{2}=\int_{0}^{\infty} \int\left|\nabla P_{t} f\right|^{2} d \mu d t
$$

since $P_{t}$ is selfajoint and $P_{t} 1=1$. Because of (2.8)

$$
\begin{aligned}
\left\|G_{2}(f)\right\|_{2}^{2} & =\int_{0}^{\infty} \int\left(P_{t} f\right)\left(d \Gamma(A) P_{t} \bar{f}\right) d \mu d t \\
& =-\frac{1}{2} \iint_{0}^{\infty} \partial_{t}\left|P_{t} f\right|^{2} d t d \mu \\
& =\frac{1}{2}\|f\|_{2}^{2}
\end{aligned}
$$

and we used selfadjointness of $d \Gamma(A)$, integration by parts and $\lim _{t \rightarrow \infty} P_{t} f$ $=0$ (pointwise), since $\int f d \mu=0$. By subadditivity of $G_{2}(f)$, we find for general $f \in L^{2}$

$$
\left\|G_{2}(f)\right\|_{2} \lesssim\|f\|_{2}
$$

and the lemma is proved.

The next step is

Lemma 3.4. Let $f \in L^{p}, p \in(1,2]$. Then

$$
\left\|G_{1}(f)\right\|_{p} \lesssim\|f\|_{p} .
$$

Proof. The case $p=2$ is already proved in the preceding lemma. Let now $p \in(1,2)$. Assume first that $f$ is real and note that it is sufficient to prove (3.7) for real $f \in \mathscr{P}$. Decompose $f$ into $f_{+}-f_{-}, f_{+}$(- $f_{-}$resp.) being its positive (negative resp.) part. Remark that $f_{ \pm}$satisfy the hypothesis of Lemma 3.1. Thus we may write

$$
\begin{aligned}
P_{t}\left|\nabla P_{t} f_{+}\right| & =(p(p-1))^{-1 / 2} P_{t}\left\{\left(P_{t} f_{+}\right)^{1-p / 2}\left[\left(-\partial_{t}-d \Gamma(A)\right)\left(P_{t} f_{+}\right)^{p}\right]^{1 / 2}\right\} \\
& \leq(p(p-1))^{-1 / 2}\left(P_{t}\left(P_{t} f_{+}\right)^{2-p}\right)^{1 / 2}\left(P_{t}\left(-\partial_{t}-d \Gamma(A)\right)\left(P_{t} f_{+}\right)^{p}\right)^{1 / 2}
\end{aligned}
$$

Let us denote

$$
g^{*}(x):=\sup _{t>0}\left|\left(P_{t} g\right)\right|(x)
$$

for pointwise defined $g$, and a lemma in [13; p. 48] states that

$$
\left\|g^{*}\right\|_{p} \lesssim\|g\|_{p}
$$

Hence, applying this to our situation

$$
G_{1}\left(f_{+}\right)^{2}(x) \leq A_{p}\left(\left(f_{+}^{*}\right)^{2-p}\right)^{*}(x) I(x)
$$


with $I(x)$ defined in (3.2) with $F(x, t)=\left(P_{t} f_{+}\right)^{p}(x)$. By Hölder's inequality and (3.9)

$$
\left\|G_{1}\left(f_{+}\right)\right\|_{p}^{p} \lesssim\left\|f_{+}\right\|_{p}^{p / 2(2-p)}\left(\int I d \mu\right)^{p / 2}
$$

Now apply Lemma 3.2 to the last factor

$$
\begin{aligned}
\int I d \mu & =\int\left(f_{+}^{p}(x)-\left(\lim _{t \rightarrow \infty}\left(P_{t} f_{+}\right)^{p}(x)\right)\right) d \mu(x) \\
& \leq \int f_{+}^{p}(x) d \mu(x)
\end{aligned}
$$

so that we have proved

$$
\left\|G_{1}\left(f_{ \pm}\right)\right\|_{p} \lesssim\left\|f_{ \pm}\right\|_{p}
$$

Since

$$
G_{1}(f+g) \leq 2^{1 / 2}\left(G_{1}(f)+G_{1}(g)\right)
$$

we find

$$
\begin{aligned}
\left\|G_{1}\left(f_{+}-f_{-}\right)\right\|_{p}^{p} & \lesssim\left\|G_{1}\left(f_{+}\right)+G_{1}\left(f_{-}\right)\right\|_{p}^{p} \\
& \lesssim \int f_{+}^{p} d \mu+\int f_{-}^{p} d \mu \\
& \lesssim \int\left(f_{+}+f_{-}\right)^{p} d \mu \\
& =\|f\|_{p}^{p},
\end{aligned}
$$

proving (3.7) in case that $f$ is real. For complex $f$, decompose it into real and imaginary part, use (3.8) and proceed similarly as for $f_{ \pm}$.

Our next lemma is

Lemma 3.5. Let $\phi, f \in \mathscr{P}, \phi$ positive. Then

$$
\int G_{2}^{2}(f) \phi d \mu \leq K\left\{\int f^{2} \phi d \mu+\int f^{*} G_{2}(f) G_{1}(\phi) d \mu\right\}
$$

for a constant $K$ and $f^{*}$ defined in (3.8).

Proof. Remark that for $p=2$ and $f \in \mathscr{P}$ equation (3.1) makes sense, except on the set of zeros of $f$, which form a set of $\mu$-measure zero. Thus (3.1) and selfadjointness of $P_{t}$ yield

$$
\int G_{2}(f)^{2} \phi d \mu=-\frac{1}{2} \iint_{0}^{\infty}\left(\left(\partial_{t}+d \Gamma(A)\right)\left(P_{t} f\right)^{2}\left(P_{t} \phi\right) d t d \mu\right.
$$


Furthermore for two polynomials $g, h$ we have the identity

$$
\left(\partial_{t}+d \Gamma(A)\right) g h=g\left(\partial_{t}+d \Gamma(A)\right) h+h\left(\partial_{t}+d \Gamma(A)\right) g-2 \nabla g \cdot \nabla h,
$$

with the notation

$$
\left.\nabla g \cdot \nabla h=\sum_{k} \lambda_{k}\left(\partial e_{k}\right) g\right)\left(\partial\left(e_{k}\right) h\right)
$$

Upon insertion

$$
\begin{aligned}
\int G_{2}(f)^{2} \phi d \mu & =\iint_{0}^{\infty}\left\{-\frac{1}{2}\left(\partial_{t}+d \Gamma(A)\right)\left(\left(P_{t} f\right)^{2}\left(P_{t} \phi\right)\right)+\nabla\left(P_{t} f\right)^{2} \cdot \nabla P_{t} \phi\right\} d t d \mu \\
& \equiv \mathrm{I}+\mathrm{II}
\end{aligned}
$$

because $\left(\partial_{t}+d \Gamma(A)\right) P_{t} \phi=0$. Then

$$
\begin{aligned}
\mathrm{I} & =-\frac{1}{2} \iint_{0}^{\infty} \partial_{t}\left(\left(P_{t} f\right)^{2} P_{t} \phi\right) d t d \mu \\
& =\frac{1}{2} \int\left(f^{2} \phi-\lim _{t \rightarrow \infty}\left(P_{t} f\right)^{2} P_{t} \phi\right) d \mu \\
& \leq \frac{1}{2} \int f^{2} \phi d \mu .
\end{aligned}
$$

It remains to show that

$$
|\mathrm{II}| \leq K \int f^{*} G_{2}(f) G_{1}(\phi) d \mu .
$$

But

$$
\begin{aligned}
|\mathrm{II}| & \leq \iint_{0}^{\infty}\left|\nabla\left(P_{t} f\right)^{2} \cdot \nabla P_{t} \phi\right| d t d \mu \\
& =2 \iint_{0}^{\infty}\left|\left(P_{t} f\right)\left(\nabla P_{t} f\right) \cdot\left(\nabla P_{t} \phi\right)\right| d t d \mu \\
& \leq 2 \int f^{*} \int_{0}^{\infty}\left|\nabla P_{t} f\right|\left|\nabla P_{t} \phi\right| d t d \mu
\end{aligned}
$$

by Schwarz' inequality on $l^{2}$. Lemma 2.4 and Corollary 2.5 yield the estimate

$$
|\mathrm{II}| \leq 2 \int f^{*} \int_{0}^{\infty}\left(P_{t / 2}\left|\nabla P_{t / 2} f\right|^{2}\right)^{1 / 2}\left(P_{t / 2}\left|\nabla P_{t / 2} \phi\right|\right) d t d \mu .
$$

Apply Schwarz' inequality for the $d t$-integration in the last expression to find

$$
|\mathrm{II}| \leq K \int f^{*} G_{2}(f) G_{1}(\phi) d \mu
$$


Now we can finally come to the

Proof of Theorem A. For $1<p \leq 2$ inequality (1.2) is already proved in Lemma 3.4. Next we prove it for $4 \leq p<\infty$, so that by (3.10) and the Marcinkiewicz interpolation theorem [12] the result follows for all $p, 1<$ $p<\infty$. Let $q$ be conjugate to $p / 2$ and $\phi \in L^{q}, \phi \geq 0$. Then

$$
\left\|G_{2}(f)\right\|_{p}^{2}=\sup _{\phi,\|\phi\|_{q} \leq 1} \int G_{2}(f)^{2} \phi d \mu
$$

and it is sufficient to take the supremum over polynomials $\phi \in \mathscr{P}$. In view of Lemma 3.5, Hölder's inequality and (3.9)

$$
\int G_{2}^{2}(f) \phi d \mu \leq \text { const. }\left\{\|f\|_{p}^{2}\|\phi\|_{q}+\|f\|_{p}\left\|G_{2}(f)\right\|_{p}\left\|G_{1}(\phi)\right\|_{q}\right\}
$$

with $q \leq 2$. Applying Lemma 3.4 to $\left\|G_{1}(\phi)\right\|_{q}$

$$
\begin{aligned}
\left\|G_{2}(f)\right\|_{p}^{2} & \lesssim \sup _{\phi,\|\phi\|_{q} \leq 1}\|\phi\|_{q}\|f\|_{p}\left(\|f\|_{p}+\left\|G_{2}(f)\right\|_{p}\right) \\
& \lesssim\|f\|_{p}\left(\|f\|_{p}+\left\|G_{2}(f)\right\|_{p}\right),
\end{aligned}
$$

from which $\left\|G_{2}(f)\right\|_{p} \lesssim\|f\|_{p}$ follows. Since $G_{1}(f) \lesssim G_{2}(f)$, we have proved (1.12) for all $p \in(1, \infty)$ and also (1.13) for $p \in[4, \infty)$. But since $G_{2}$ is subadditive and $\left\|G_{2}(f)\right\|_{2} \leq 2^{-1 / 2}\|f\|_{2}$, (1.13) holds for $p \in[2, \infty)$ by the Marcinkiewicz interpolation theorem. (1.14) follows from (1.13) and the equality (3.6) $\left\|G_{2}(f)\right\|_{2}=2^{-1 / 2}\|f\|_{2}$ if $\int f d \mu=0$, by duality.

\section{§4. LPS-Inequalities: Elliptic Case}

As mentioned in the introduction, this case is quite parallel to the parabolic one and only the major steps of the proof of Theorem B are indicated.

Firstly, equation (3.1) is now replaced by

$$
\left(\partial_{t}^{2}-d \Gamma(A)\right)\left(Q_{t} f\right)^{p}=p(p-1)\left(Q_{t} f\right)^{p-2}\left[\left(\partial_{t} Q_{t} f\right)^{2}+\left|\nabla Q_{t} f\right|^{2}\right]
$$

for $p>1, t>0$ and $f$ as in the hypothesis of Lemma 3.1.

The function $I(x)$, eq. (3.2), has to be defined in this case by

$$
I(x):=\int_{0}^{\infty} t\left(\partial_{t}^{2}-d \Gamma(A)\right) F(x, t) d t,
$$

and (3.3) holds again, if $t\left\|\partial_{t} F(x, t)\right\|_{2} \rightarrow 0$ as $t \rightarrow \infty, t \rightarrow 0$. 
In the same way as Lemma 3.3 (in fact a bit simpler), we can prove the bound

$$
\left\|G_{3}(f)\right\|_{p} \lesssim\|f\|_{2}
$$

for all $p \in(1,2], f \in L^{2}$, this time using the equality

$$
\left\|G_{3}(f)\right\|_{2}=2^{-1 / 2}\|f\|_{2}
$$

for $f \in L^{2}$ with $\int f d \mu=0$, since, first

$$
\begin{aligned}
\iint_{0}^{\infty} t\left|\partial_{t} Q_{t} f\right|^{2} d t d \mu & =\iint_{0}^{\infty} t\left\{\frac{1}{2} \partial_{t}\left|Q_{t} f\right|^{2}-\left(\partial_{t}^{2} Q_{t} f\right)\left(Q_{t} \bar{f}\right)\right\} d t d \mu \\
& =\frac{1}{2}\|f\|_{2}^{2}-\iint_{0}^{\infty} t\left|\partial_{t} Q_{t} f\right|^{2} d t d \mu
\end{aligned}
$$

or $\iint_{0}^{\infty} t\left|\partial_{t} Q_{t} f\right|^{2} d t d \mu=\frac{1}{4}\|f\|_{2}^{2}$ and second

$$
\begin{aligned}
\iint_{0}^{\infty} t\left|\nabla Q_{t} f\right|^{2} d t d \mu & =\iint_{0}^{\infty} t\left(d \Gamma(A) Q_{t} f\right)\left(Q_{t} \bar{f}\right) d t d \mu \\
& =\iint_{0}^{\infty} t\left|\partial_{t} Q_{t} f\right|^{2} d t d \mu \\
& =\frac{1}{4}\|f\|_{2}^{2}
\end{aligned}
$$

and we have used Fubini's theorem, selfadjointness of $d \Gamma(A)$ and integration by parts.

Next, analogous to Lemma 3.4 we can prove

Lemma 4.1. Let $f \in L^{p}, p \in(1,2]$, then $G_{3}(f)$ is in $L^{p}$ too and

$$
\left\|G_{3}(f)\right\|_{p} \lesssim\|f\|_{p}
$$

and also

LEMMA 4.2. For polynomials $f$ and $\phi \geq 0$

$$
\int G_{3}(f)^{2} \phi d \mu \leq K\left\{\int f^{2} \phi d \mu+\int f^{* *} G_{3}(f) G_{3}(\phi) d \mu\right\}
$$

for some constant $K$ and

$$
f^{* *}(x):=\sup _{t>0}\left|Q_{t} f\right|(x)
$$

is proved as the corresponding lemma in section 3, in this case somewhat easier. 
Sketch of the proof of Theorem B. Use Lemmas 4.1, 4.2, subadditivity of $G_{3}$ and the Marcinkiewicz interpolation theorem to prove (1.15) for all $p \in(1, \infty)$. To obtain the converse inequality for $f \in L^{p}$ with $\int f d \mu=0$, use equality (4.4), inequality (1.15) and duality.

Let us define

$$
\begin{aligned}
& G_{4}(f):=\left(\int_{0}^{\infty} t\left|\partial_{t} Q_{t} f\right|^{2} d t\right)^{1 / 2} \\
& G_{5}(f):=\left(\int_{0}^{\infty} t\left|\nabla Q_{t} f\right|^{2} d t\right)^{1 / 2}
\end{aligned}
$$

then we have immediately from the preceding arguments the following

TheOREM 4.3. If $f \in L^{p}, p \in(1, \infty)$, then so are $G_{4}(f)$ and $G_{5}(f)$ and their $L^{p}$-norms are bounded by $A_{p}\|f\|_{p}$, for some constant $A_{p}$. If in addition $\int f d \mu=0$, then also

$$
\|f\|_{p} \lesssim\left\|G_{i}(f)\right\|_{p}, \quad i=4,5
$$

Proof. The first statement follows trivially from Theorem B and the second from the proof of equality (4.4) and duality.

Let us conclude this paper in showing a slight generalization of Theorem 4.3 for $G_{4}$.

THEOREM 4.4. Let $\left\{f_{k}, k \in Z_{+}\right\}$be a sequence of functions $f_{k} \in L^{p}, 1<p$ $<\infty$ and suppose that $\left(\sum_{k} f_{k}^{2}\right)^{1 / 2} \in L^{p}$. Then also $\left(\sum_{k} G_{4}\left(f_{k}\right)^{2}\right)^{1 / 2} \in L^{p}$ and

$$
\left\|\left(\sum_{k} G_{4}\left(f_{k}\right)^{2}\right)^{1 / 2}\right\|_{p} \lesssim\left\|\left(\sum_{k} f_{k}^{2}\right)^{1 / 2}\right\|_{p}
$$

holds. Furthermore, if for all $k \int f_{k} d \mu=0$, then

$$
\left\|\left(\sum_{k} f_{k}^{2}\right)^{1 / 2}\right\|_{p} \lesssim\left\|\left(\sum_{k} G_{4}(f)^{2}\right)^{1 / 2}\right\|_{p}
$$

Proof. Theorem 4.4 follows from Theorem 4.3 and the standard trick to employ Rademacher functions [1,12]. Let $\left\{\boldsymbol{r}_{k}, k \in \boldsymbol{Z}_{+}\right\}$be a system of Rademacher functions on $[0,1]$, and let $I$ be a finite subset of $Z_{+}$. Consider the $L^{p}$-function

$$
F(u)=\sum_{k \in I} r_{k}(u) f_{k}, \quad u \in[0,1] .
$$

Apply Theorem 4.3 to $F(u)$ and integrate: 


$$
\int_{0}^{1}\left\|G_{4}(F(u))\right\|_{p}^{p} d u \lesssim \int_{0}^{1}\|F(u)\|_{p}^{p} d u
$$

By Khintchin's inequalities (cf. $[1,12]$ ) on $l^{2}$ the righthand side of (4.12) is equivalent to

$$
\left\|\left(\sum_{k \in I} f_{k}^{2}\right)^{1 / 2}\right\|_{p}^{p},
$$

while the lefthand side of (4.12) is equivalent to

$$
\text { I) }\left(\sum_{k \in I} G_{4}\left(f_{k}\right)^{2}\right)^{1 / 2} \|_{p}^{p}
$$

again by Khintchin's inequalities, this time on $L^{2}\left(\boldsymbol{R}_{+}, t d t\right)$. Increasing $I$ to $Z_{+},(4.10)$ is proved. The converse inequality (4.11) follows by the same argument.

Remark. It is obvious, that similar generalizations can be found for the other LPS-inequalities.

\section{REFERENCES}

[1] Burkholder, D. L., A geometrical characterization of Banach spaces in which martingale differences are unconditional, Ann. Probab., 9 (1981), 997-1011.

[2 ] Cook, J., The mathematics of second quantization, Trans. Amer. Math. Soc., 74 (1953), 222-245.

[ 3 ] Gel'fand, I. M., Vilenkin, N. Y., Generalized functions, vol. 4. New York, London: Academic Press 1964.

[ 4 ] Gröbner, W., Hofreiter, N., Integraltafel, Zweiter Teil. Wien und Innsbruck: Springer-Verlag 1958.

[ 5 ] Hida, T., Brownian motion, Berlin, Heidelberg, New York: Springer 1980.

[6] Meyer, P. A., Démonstration probabiliste de certaines inégalités de LittlewoodPaley, Séminaire de probabilités X, ed. Azéma, J., Yor, M. Berlin, Heidelberg, New York: Springer 1976.

[7] - Quelques resultats analytiques sur le semigroupe d'Ornstein-Uhlenbeck en dimension infinie, In: Theory and application of random fields (ed. by G. Kallianpur), Berlin, Heidelberg, New York: Springer 1983.

[ 8 ] Nelson, E., The free Markoff field, J. Funct. Anal., 12 (1973), 211-227.

[9] —- Probability theory and Euclidean quantum field theory, In Constructive quantum field theory (ed. by G. Velo and A. Wightman), Berlin, Heidelberg, New York: Springer 1973.

[10] Reed, M., Simon, B., Methods in mathematical physics I, New York, London: Academic Press 1975.

[11] Simon, B., The $P(\phi)_{2}$ Euclidean (quantum) field theory, Princeton: Princeton University Press 1970.

[12] Stein, E. M., Singular integrals and differentiability properties of functions, Princeton: Princeton University Press 1970.

[13] — Topics in harmonic analysis, Princeton: Princeton University Press 1970. 
Department of Mathematics

Nagoya University

Nagoya 464, Japan

and

Department of Mathematics, MA7-1

Technical University Berlin

Strasse d. 1\%. Juni 135

$D-1000, B$ erlin 12 